\title{
Correction to: Does timing in ICSI cycle affect oocyte quality and reproductive outcomes? A prospective study
}

\author{
Elham Azizi ${ }^{1}$ - Mohammad Naji ${ }^{2} \cdot$ Hamid Nazarian $^{1} \cdot$ Saghar Salehpour $^{3} \cdot$ Maryam Karimi $^{3} \cdot$ Nasrin Borumandnia $^{2}$. \\ Zahra Shams Mofarahe ${ }^{4}$ (1)
}

Published online: 29 May 2020

(c) Springer-Verlag GmbH Germany, part of Springer Nature 2020

\section{Correction to: Archives of Gynecology and Obstetrics https://doi.org/10.1007/s00404-020-05555-w}

In the original article published, the values given in the variables are incorrect. The correct values are given in the Tables 1, 2, 3 below. The original article has been corrected s00404-020-05555-w.

Zahra Shams Mofarahe

z_shams@sbmu.ac.ir

Elham Azizi

Elh.4329@yahoo.com

Mohammad Naji

Naji_m_f@yahoo.com

Hamid Nazarian

Hamid.Nazarian@gmail.com

Saghar Salehpour

saghar.salehpour2014@gmail.com

Maryam Karimi

Karimi_m_1985@yahoo.com
Nasrin Borumandnia borumand.n@gmail.com

1 Department of Biology and Anatomical Sciences, School of Medicine, Shahid Beheshti University of Medical Sciences, Tehran, Iran

2 Urology and Nephrology Research Center (UNRC), Shahid Beheshti University of Medical Sciences, Tehran, Iran

3 IVF Center, Taleghani Hospital, Shahid Beheshti University of Medical Sciences, Tehran, Iran

4 Men's Health and Reproductive Health Research Center (MHRHRC), Shahid Beheshti University of Medical Sciences, Tehran, Iran 
Table 1 Demographic and ICSI cycle characteristics of patients

\begin{tabular}{|c|c|c|c|c|c|c|c|c|c|}
\hline \multirow[t]{3}{*}{ Variable } & \multicolumn{4}{|c|}{ HCG-OPU $\leq 36$ h $(n=264)$} & \multicolumn{4}{|c|}{ HCG-OPU > 36 h $(n=349)$} & \multirow[t]{3}{*}{$P$ value } \\
\hline & \multicolumn{2}{|c|}{ OPU-DN $\leq 2 \mathrm{~h}(n=141)$} & \multicolumn{2}{|c|}{ OPU $-\mathrm{DN}>2 \mathrm{~h}(n=123)$} & \multicolumn{2}{|c|}{ OPU-DN $\leq 2 \mathrm{~h}(n=180)$} & \multicolumn{2}{|c|}{ OPU -DN > 2 h $(n=169)$} & \\
\hline & $\begin{array}{l}\mathrm{DN}-\mathrm{ICSI} \leq 2 \mathrm{~h} \\
(n=101)\end{array}$ & $\begin{array}{l}\text { DN-ICSI }>2 \mathrm{~h} \\
(n=40)\end{array}$ & $\begin{array}{l}\mathrm{DN}-\mathrm{ICSI} \leq 2 \mathrm{~h} \\
(n=75)\end{array}$ & $\begin{array}{l}\text { DN-ICSI }>2 \mathrm{~h} \\
(n=48)\end{array}$ & $\begin{array}{l}\mathrm{DN}-\mathrm{ICSI} \leq 2 \mathrm{~h} \\
(n=119)\end{array}$ & $\begin{array}{l}\text { DN-ICSI }>2 \mathrm{~h} \\
(n=61)\end{array}$ & $\begin{array}{l}\mathrm{DN}-\mathrm{ICSI} \leq 2 \mathrm{~h} \\
(n=104)\end{array}$ & $\begin{array}{l}\text { DN-ICSI }>2 \mathrm{~h} \\
(n=65)\end{array}$ & \\
\hline $\begin{array}{l}\text { hCG-OPU } \\
\text { interval }(\mathrm{h}) \\
\text { mean } \pm \mathrm{SD}, \\
\text { range }\end{array}$ & $\begin{array}{l}35.7 \pm 0.3 \\
35.0-36.0\end{array}$ & $\begin{array}{l}35.8 \pm 0.2 \\
35.0-36.0\end{array}$ & $\begin{array}{l}35.7 \pm 0.3 \\
35.0-36.0\end{array}$ & $\begin{array}{l}35.7 \pm 0.2 \\
35.1-36.0\end{array}$ & $\begin{array}{l}36.8 \pm 0.8 \\
36.1-41.7\end{array}$ & $\begin{array}{l}36.7 \pm 0.5 \\
36.1-38.2\end{array}$ & $\begin{array}{l}36.9 \pm 0.8 \\
36.1-40.3\end{array}$ & $\begin{array}{l}36.7 \pm 0.5 \\
36.1-38.7\end{array}$ & \\
\hline $\begin{array}{l}\text { OPU-denu- } \\
\text { dation }(\mathrm{h}) \text {, } \\
\text { mean } \pm \mathrm{SD} \text {, } \\
\text { range }\end{array}$ & $\begin{array}{r}1.5 \pm 0.4 \\
0.5-2.0\end{array}$ & $\begin{array}{r}1.4 \pm 0.4 \\
0.6-2.0\end{array}$ & $\begin{array}{r}3.2 \pm 1.1 \\
2.1-5.8\end{array}$ & $\begin{array}{r}2.9 \pm 0.9 \\
2.1-6.0\end{array}$ & $\begin{array}{r}1.3 \pm 0.5 \\
0.2-2.0\end{array}$ & $\begin{array}{r}1.4 \pm 0.4 \\
0.2-2.0\end{array}$ & $\begin{array}{r}3.4 \pm 1.1 \\
2.1-6.0\end{array}$ & $\begin{array}{r}3.2 \pm 0.8 \\
2.1-5.5\end{array}$ & \\
\hline $\begin{array}{l}\text { Denudation- } \\
\text { ICSI }(h), \\
\text { mean } \pm S D, \\
\text { range }\end{array}$ & $\begin{array}{l}1.1 \pm 0.5,0.1 \\
\quad-2.0\end{array}$ & $\begin{array}{r}3.3 \pm 1.0 \\
2.2-5.8\end{array}$ & $\begin{array}{r}1.0 \pm 0.5 \\
0.1-2.0\end{array}$ & $\begin{array}{r}3.6 \pm 1.2 \\
2.3-6.0\end{array}$ & $\begin{array}{r}1.2 \pm 0.6 \\
0.1-2.0\end{array}$ & $\begin{array}{r}3.4 \pm 1.1 \\
2.1-6.0\end{array}$ & $\begin{array}{r}1.1 \pm 0.5 \\
0.1-2.0\end{array}$ & $\begin{array}{r}3.0 \pm 0.8 \\
2.1-4.8\end{array}$ & \\
\hline Female age (Y) & $33.6 \pm 6.1$ & $33.6 \pm 6.5$ & $31.2 \pm 6.0^{\mathrm{a}}$ & $32.7 \pm 5.8$ & $34.5 \pm 6.5^{\mathrm{ab}}$ & $34.4 \pm 5.9$ & $31.8 \pm 5.8^{\mathrm{b}}$ & $32.3 \pm 5.5$ & 0.003 \\
\hline BMI $\left(\mathrm{kg} / \mathrm{m}^{2}\right)$ & $26.0 \pm 4.1$ & $25.7 \pm 3.8$ & $26.0 \pm 3.3$ & $26.2 \pm 3.9$ & $25.4 \pm 4.1$ & $24.5 \pm 3.4$ & $25.0 \pm 3.9$ & $26.7 \pm 3.6$ & 0.02 \\
\hline FSH (IU/mL) & $6.5 \pm 4.2$ & $5.9 \pm 2.9$ & $5.8 \pm 2.3$ & $6.7 \pm 4.8$ & $6.1 \pm 4.6$ & $5.1 \pm 2.5$ & $6.3 \pm 4.2$ & $7.1 \pm 7.9$ & 0.9 \\
\hline $\mathrm{AMH}(\mathrm{ng} / \mathrm{mL})$ & $3.2 \pm 2.9$ & $3.8 \pm 3.4$ & $3.8 \pm 3.9$ & $3.8 \pm 3.9$ & $3.4 \pm 3.4$ & $4.7 \pm 4.7$ & $3.7 \pm 4.1$ & $4.5 \pm 4.0$ & 0.3 \\
\hline $\begin{array}{l}\text { Primary infertil- } \\
\text { ity }(n)\end{array}$ & $74(73.3)$ & $36(90.0)$ & $62(82.7)$ & $42(87.5)$ & $90(75.6)$ & $49(80.3)$ & $88(84.6)$ & $56(86.1)$ & 0.1 \\
\hline $\begin{array}{l}\text { Secondary } \\
\quad \text { infertility }(n)\end{array}$ & $27(26.7)$ & $4(10.0)$ & $13(17.3)$ & $6(12.5)$ & $29(24.4)$ & $12(19.7)$ & $16(15.4)$ & $9(13.9)$ & \\
\hline $\begin{array}{l}\text { Infertility dura- } \\
\text { tion (year) }\end{array}$ & $5.3 \pm 4.6$ & $7.2 \pm 6.7$ & $4.8 \pm 3.7$ & $4.6 \pm 3.6$ & $5.6 \pm 4.7$ & $5.9 \pm 4.7$ & $5.3 \pm 4.8$ & $5.2 \pm 4.3$ & 0.6 \\
\hline \multicolumn{10}{|l|}{$\begin{array}{l}\text { No. of previous } \\
\text { ICSI cycles }\end{array}$} \\
\hline 0 & $64(63.4)$ & $28(70.0)$ & $52(69.3)$ & $30(62.5)$ & $74(62.1)$ & $33(54.1)$ & $75(72.1)$ & $45(69.2)$ & 0.4 \\
\hline 1 & 27 (26.7) & $8(20.0)$ & $14(18.7)$ & $13(27.1)$ & $27(22.7)$ & $13(21.3)$ & $17(16.3)$ & $10(15.4)$ & \\
\hline 2 & $8(7.9)$ & $2(5.0)$ & $7(9.3)$ & $5(10.4)$ & $14(11.8)$ & $10(16.4)$ & $6(5.8)$ & $6(9.2)$ & \\
\hline$\geq 3$ & $2(2.0)$ & $2(5.0)$ & $2(2.7)$ & $0(0)$ & $4(3.4)$ & $5(8.2)$ & $6(5.8)$ & $4(6.2)$ & \\
\hline \multicolumn{10}{|l|}{$\begin{array}{l}\text { Cause of infer- } \\
\text { tility }(n)\end{array}$} \\
\hline Male factor & $47(46.5)$ & $24(60.0)$ & $39(52.0)$ & $21(43.7)$ & $41(34.4)$ & $27(44.3)$ & $49(47.1)$ & $29(44.6)$ & 0.1 \\
\hline AMA & 12 (11.9) & $2(5.0)$ & $3(4.0)$ & $6(12.5)$ & $13(10.9)$ & 7 (11.5) & $6(5.8)$ & $7(10.8)$ & 0.4 \\
\hline DOR & $21(20.8)$ & 7 (17.5) & $6(8.0)$ & $6(12.5)$ & $26(21.8)$ & $6(9.8)$ & $8(7.7)$ & $4(6.1)$ & 0.004 \\
\hline PCOS & $25(24.7)$ & $11(27.5)$ & $16(21.3)$ & $16(33.3)$ & $20(16.8)$ & $14(22.9)$ & $22(21.1)$ & $18(27.7)$ & 0.4 \\
\hline Endometriosis & $4(4.0)$ & $1(2.5)$ & $3(4.0)$ & $2(4.2)$ & $8(6.7)$ & $0(0)$ & $4(3.8)$ & $0(0)$ & 0.3 \\
\hline RIF & $7(6.9)$ & $4(10.0)$ & $6(8.0)$ & $2(4.2)$ & $7(5.9)$ & $10(16.4)$ & $6(5.8)$ & $9(13.8)$ & 0.1 \\
\hline Uterine & $7(6.9)$ & $1(2.5)$ & $5(6.7)$ & $2(4.2)$ & $12(10.1)$ & $4(6.6)$ & $6(5.8)$ & $6(9.2)$ & 0.7 \\
\hline Others & 14 (13.9) & $5(12.5)$ & $10(13.3)$ & $5(10.4)$ & $17(14.3)$ & $9(14.7)$ & $18(17.3)$ & $10(15.4)$ & 0.9 \\
\hline \multicolumn{10}{|l|}{$\begin{array}{l}\text { Stimulation } \\
\quad \text { protocol }(n)\end{array}$} \\
\hline Antagonist & $97(96.0)$ & 37 (92.5) & $71(94.7)$ & 45 (93.7) & $110(92.4)$ & $56(91.8)$ & $97(93.3)$ & $63(96.9)$ & 0.8 \\
\hline Agonist & $4(4.0)$ & $3(7.5)$ & $4(5.3)$ & $3(6.3)$ & $9(7.6)$ & $5(8.2)$ & $7(6.7)$ & $2(3.1)$ & \\
\hline $\begin{array}{c}\text { Total dose of } \\
\text { gonadotro- } \\
\text { phins (IU) }\end{array}$ & $2406.0 \pm 1384.3$ & $2815.6 \pm 1435.2$ & $2234.7 \pm 956.7$ & $1909.1 \pm 994.1$ & $2636.7 \pm 1310.7$ & $2581.4 \pm 1548.7$ & $2526.8 \pm 1328.6$ & $2076.9 \pm 908.0$ & 0.09 \\
\hline $\begin{array}{l}\text { No. of embryos } \\
\text { transferred }\end{array}$ & $1.7 \pm 0.5$ & $1.7 \pm 0.6$ & $1.8 \pm 0.5$ & $1.7 \pm 0.4$ & $1.8 \pm 0.6$ & $1.9 \pm 0.5$ & $1.8 \pm 0.5$ & $1.7 \pm 0.5$ & 0.7 \\
\hline
\end{tabular}


Table 2 Characteristics of oocytes in ICSI cycles

\begin{tabular}{|c|c|c|c|c|c|c|c|c|c|}
\hline \multirow[t]{3}{*}{ Variable } & \multicolumn{4}{|c|}{ HCG-OPU $\leq 36 \mathrm{~h}(n=264)$} & \multicolumn{4}{|c|}{ HCG-OPU > 36 h $(n=349)$} & \multirow[t]{3}{*}{$P$ value } \\
\hline & \multicolumn{2}{|c|}{ OPU-DN $\leq 2 \mathrm{~h}(n=141)$} & \multicolumn{2}{|c|}{ OPU $-\mathrm{DN}>2 \mathrm{~h}(n=123)$} & \multicolumn{2}{|c|}{ OPU-DN $\leq 2 \mathrm{~h}(n=180)$} & \multicolumn{2}{|c|}{ OPU-DN $>2 \mathrm{~h}(n=169)$} & \\
\hline & $\begin{array}{l}\mathrm{DN}- \\
\mathrm{ICSI} \leq 2 \mathrm{~h} \\
(n=101)\end{array}$ & $\begin{array}{l}\mathrm{DN}- \\
\mathrm{ICSI}>2 \mathrm{~h} \\
(n=40)\end{array}$ & $\begin{array}{l}\mathrm{DN}- \\
\mathrm{ICSI} \leq 2 \mathrm{~h} \\
(n=75)\end{array}$ & $\begin{array}{l}\mathrm{DN}- \\
\mathrm{ICSI}>2 \mathrm{~h} \\
(n=48)\end{array}$ & $\begin{array}{l}\mathrm{DN}- \\
\mathrm{ICSI} \leq 2 \mathrm{~h} \\
(n=119)\end{array}$ & $\begin{array}{l}\text { DN- } \\
\text { ICSI }>2 \mathrm{~h} \\
(n=61)\end{array}$ & $\begin{array}{l}\mathrm{DN}- \\
\mathrm{ICSI} \leq 2 \mathrm{~h} \\
(n=104)\end{array}$ & $\begin{array}{l}\text { DN-ICSI }>2 \mathrm{~h} \\
(n=65)\end{array}$ & \\
\hline $\begin{array}{l}\text { Total number } \\
\text { of oocytes } \\
\text { aspirated } \\
(\text { mean } \pm \text { SD) }\end{array}$ & $\begin{array}{l}717 \\
\quad(7.1 \pm 4.5)\end{array}$ & $\begin{array}{l}330 \\
\quad(8.2 \pm 5.3)\end{array}$ & $\begin{array}{l}654 \\
\quad(8.7 \pm 5.7)\end{array}$ & $\begin{array}{l}461 \\
\quad(9.6 \pm 6.8)\end{array}$ & $\begin{array}{l}805 \\
\quad(6.8 \pm 5.7)^{\mathrm{a}}\end{array}$ & $\begin{array}{l}523 \\
\quad(8.6 \pm 5.1)\end{array}$ & $\begin{array}{l}959 \\
\quad(9.2 \pm 7.3)\end{array}$ & $608(9.3 \pm 5.0)^{\mathrm{a}}$ & 0.0006 \\
\hline $\begin{array}{c}\text { Metaphase II } \\
\text { oocytes }(n)\end{array}$ & $5.7 \pm 4.0$ & $6.5 \pm 4.7$ & $6.8 \pm 4.6$ & $7.5 \pm 5.1^{\mathrm{a}}$ & $5.3 \pm 5.0^{\mathrm{ab}}$ & $6.6 \pm 4.6$ & $6.5 \pm 5.5$ & $7.0 \pm 4.1^{\mathrm{b}}$ & 0.004 \\
\hline $\begin{array}{l}\text { Ratio of } \\
\text { metaphase II } \\
\text { oocytes/total } \\
\text { oocytes }\end{array}$ & $0.8 \pm 0.2$ & $0.8 \pm 0.2$ & $0.8 \pm 0.2$ & $0.8 \pm 0.2$ & $0.8 \pm 0.2$ & $0.8 \pm 0.2$ & $0.7 \pm 0.2$ & $0.8 \pm 0.2$ & 0.05 \\
\hline $\begin{array}{l}\text { Cytoplasm } \\
\text { granulation } \\
(\%)\end{array}$ & $23.7 \pm 37.5$ & $25.1 \pm 40.1$ & $31.3 \pm 39.2$ & $26.1 \pm 40.0$ & $26.8 \pm 39.4$ & $26.9 \pm 38.0$ & $36.5 \pm 40.6$ & $20.0 \pm 35.9$ & 0.09 \\
\hline $\begin{array}{l}\text { Central } \\
\text { granulation } \\
\text { of cytoplasm } \\
(\%)\end{array}$ & $7.4 \pm 24.0$ & $14.5 \pm 33.5$ & $13.6 \pm 29.0$ & $12.0 \pm 29.8$ & $13.0 \pm 30.5$ & $9.2 \pm 24.3$ & $9.6 \pm 25.0$ & $7.3 \pm 22.1$ & 0.7 \\
\hline $\begin{array}{l}\text { Vacuole in } \\
\text { cytoplasm } \\
(\%)\end{array}$ & $6.2 \pm 20.8$ & $4.0 \pm 16.5$ & $2.0 \pm 8.7$ & $1.3 \pm 5.6$ & $2.0 \pm 10.5$ & $5.5 \pm 15.7$ & $5.2 \pm 17.3$ & $8.8 \pm 21.4$ & 0.05 \\
\hline $\begin{array}{l}\text { SER clustering } \\
(\%)\end{array}$ & $0.6 \pm 3.4$ & $2.6 \pm 15.8$ & $0.8 \pm 7.2$ & $2.4 \pm 14.6$ & $3.2 \pm 12.2$ & $5.1 \pm 19.0$ & $2.0 \pm 11.7$ & $2.0 \pm 10.7$ & 0.2 \\
\hline $\begin{array}{l}\text { Inclusion body } \\
(\%)\end{array}$ & $20.4 \pm 34.9$ & $9.6 \pm 25.0$ & $19.9 \pm 35.5$ & $13.8 \pm 29.2$ & $15.7 \pm 30.0$ & $24.5 \pm 34.2$ & $25.8 \pm 37.3$ & $28.2 \pm 37.6$ & 0.01 \\
\hline $\begin{array}{l}\text { Amorph } \\
\text { oocyte (\%) }\end{array}$ & $2.1 \pm 7.5$ & $4.9 \pm 16.9$ & $6.5 \pm 16.1$ & $4.1 \pm 9.0$ & $5.0 \pm 17.5$ & $2.3 \pm 7.0$ & $5.9 \pm 18.5$ & $3.5 \pm 15.1$ & 0.2 \\
\hline $\begin{array}{r}\text { Oval shaped } \\
\text { oocyte }(\%)\end{array}$ & $0.8 \pm 4.9$ & $0.1 \pm 0.8$ & $1.7 \pm 7.2$ & $0.7 \pm 4.8$ & $2.4 \pm 12.8$ & $0.7 \pm 3.0$ & $0.9 \pm 5.7$ & $1.6 \pm 6.1$ & 0.7 \\
\hline $\begin{array}{l}\text { Dark oocyte } \\
(\%)\end{array}$ & 0 & 0 & $0.1 \pm 1.1$ & $0.3 \pm 2.1$ & $0.6 \pm 4.9$ & 0 & 0 & 0 & 0.2 \\
\hline $\begin{array}{l}\text { Extended PVS } \\
(\%)\end{array}$ & $30.7 \pm 39.5$ & $35.8 \pm 42.1$ & $26.7 \pm 35.6$ & $23.2 \pm 34.3$ & $39.8 \pm 42.5$ & $29.6 \pm 38.7$ & $39.8 \pm 41.5$ & $27.1 \pm 39.1$ & 0.05 \\
\hline $\begin{array}{c}\text { Granulated } \\
\text { PVS (\%) }\end{array}$ & $19.7 \pm 36.5$ & $23.1 \pm 37.4$ & $21.4 \pm 35.0$ & $19.5 \pm 34.1$ & $24.0 \pm 38.9$ & $22.5 \pm 34.7$ & $29.2 \pm 38.6$ & $24.2 \pm 39.1$ & 0.4 \\
\hline $\begin{array}{l}\text { Multiple } \\
\text { fragmented } \\
\text { PB (\%) }\end{array}$ & $47.2 \pm 41.7$ & $34.5 \pm 38.9$ & $45.6 \pm 39.9$ & $37.8 \pm 39.9$ & $37.0 \pm 39.7$ & $50.0 \pm 38.7$ & $43.5 \pm 40.2$ & $50.3 \pm 39.7$ & 0.1 \\
\hline Thin ZP (\%) & $2.6 \pm 14.4$ & $7.1 \pm 21.4$ & $5.1 \pm 18.7$ & $3.0 \pm 14.2$ & $3.4 \pm 17.4$ & $5.10 \pm 17.6$ & $0.2 \pm 1.6$ & $2.5 \pm 13.8$ & 0.1 \\
\hline Thick ZP (\%) & $1.9 \pm 11.6$ & $4.4 \pm 19.5$ & $1.8 \pm 12.1$ & $2.9 \pm 15.1$ & $3.9 \pm 18.5$ & 0 & $0.2 \pm 2.5$ & $3.9 \pm 18.5$ & 0.4 \\
\hline $\begin{array}{l}\text { Double frag- } \\
\text { mented PB } \\
(\%)\end{array}$ & $0.4 \pm 3.3$ & $1.6 \pm 9.5$ & $0.5 \pm 4.6$ & $0.3 \pm 1.5$ & $0.7 \pm 5.2$ & $0.5 \pm 3.7$ & $1.6 \pm 10.7$ & $1.8 \pm 12.5$ & 0.9 \\
\hline Huge PB (\%) & $0^{\mathrm{a}}$ & 0 & $0^{\mathrm{b}}$ & $0^{\mathrm{c}}$ & $0.04 \pm 0.38^{\mathrm{d}}$ & $0.18 \pm 1.42$ & $0^{\mathrm{e}}$ & $2.24 \pm 12.78^{\text {abcde }}$ & 0.002 \\
\hline AOQI & $1.6 \pm 1.1$ & $1.7 \pm 1.3$ & $1.8 \pm 1.1$ & $1.5 \pm 1.0$ & $1.8 \pm 1.1$ & $1.8 \pm 1.0$ & $2.0 \pm 1.2$ & $1.8 \pm 1.3$ & 0.3 \\
\hline
\end{tabular}


Table 3 Outcomes of ICSI cycles

\begin{tabular}{|c|c|c|c|c|c|c|c|c|c|}
\hline \multirow[t]{3}{*}{ Variable } & \multicolumn{4}{|c|}{ HCG-OPU $\leq 36$ h $(n=264)$} & \multicolumn{4}{|c|}{ HCG-OPU > 36 h $(n=349)$} & \multirow[t]{3}{*}{$P$ value } \\
\hline & \multicolumn{2}{|c|}{ OPU-DN $\leq 2 \mathrm{~h}(n=141)$} & \multicolumn{2}{|c|}{ OPU $-\mathrm{DN}>2 \mathrm{~h}(n=123)$} & \multicolumn{2}{|c|}{ OPU-DN $\leq 2 \mathrm{~h}(n=180)$} & \multicolumn{2}{|c|}{ OPU-DN > 2 h $(n=169)$} & \\
\hline & $\begin{array}{l}\mathrm{DN}- \\
\mathrm{ICSI} \leq 2 \mathrm{~h} \\
(n=101)\end{array}$ & $\begin{array}{l}\text { DN- } \\
\text { ICSI }>2 \mathrm{~h} \\
(n=40)\end{array}$ & $\begin{array}{l}\mathrm{DN}- \\
\mathrm{ICSI} \leq 2 \mathrm{~h} \\
(n=75)\end{array}$ & $\begin{array}{l}\text { DN- } \\
\text { ICSI }>2 \mathrm{~h} \\
(n=48)\end{array}$ & $\begin{array}{l}\mathrm{DN}- \\
\mathrm{ICSI} \leq 2 \mathrm{~h} \\
(n=119)\end{array}$ & $\begin{array}{l}\text { DN- } \\
\text { ICSI }>2 \mathrm{~h} \\
(n=61)\end{array}$ & $\begin{array}{l}\mathrm{DN}- \\
\mathrm{ICSI} \leq 2 \mathrm{~h} \\
(n=104)\end{array}$ & $\begin{array}{l}\text { DN- } \\
\text { ICSI }>2 \mathrm{~h} \\
(n=65)\end{array}$ & \\
\hline $\begin{array}{l}\text { Fertilization } \\
\text { rate }(\%)\end{array}$ & $70.2 \pm 29.1$ & $69.3 \pm 26.1$ & $71.9 \pm 23.2$ & $72.6 \pm 24.2$ & $73.5 \pm 29.9$ & $69.2 \pm 28.4$ & $67.9 \pm 29.6$ & $71.5 \pm 25.7$ & 0.7 \\
\hline $\begin{array}{l}\text { Cleavage rate } \\
(\%)\end{array}$ & $91.1 \pm 24.0$ & $96.3 \pm 16.5$ & $93.6 \pm 19.5$ & $94.6 \pm 17.6$ & $86.2 \pm 31.5$ & $95.9 \pm 15.4$ & $92.2 \pm 22.4$ & $90.0 \pm 27.8$ & 0.5 \\
\hline $\begin{array}{l}\text { Biochemical } \\
\text { pregnancy } \\
\text { rate per } \\
\text { transfer }(\%)\end{array}$ & 31.3 & 38.2 & 34.4 & 18.6 & 33.0 & 30.0 & 28.6 & 25.9 & 0.6 \\
\hline $\begin{array}{l}\text { Clinical preg- } \\
\text { nancy rate } \\
\text { per transfer } \\
(\%)\end{array}$ & 29.6 & 26.5 & 34.4 & 16.3 & 27.7 & 28.0 & 25.6 & 24.1 & 0.6 \\
\hline
\end{tabular}

Publisher's Note Springer Nature remains neutral with regard to jurisdictional claims in published maps and institutional affiliations. 